\title{
A Steganography Approach for Printed Image Based on Image Complexity and Template Matching
}

\author{
Caiyin Wang ${ }^{1,2, *}$ and Chao $\mathrm{Li}^{2}$
}

\author{
${ }^{I}$ Faculty of Electronic Information and Electrical Engineering, Dalian University of Technology, Dalian, Liaoning \\ Province 116024, P.R. China \\ ${ }^{2}$ School of Light Industry and Chemical Engineering, Dalian Polytechnic University, Dalian, Liaoning Province \\ 116034, P.R. China
}

\begin{abstract}
After a digital image is discretized, printed and rescanned, it is usually filtered, rotated, scaled, cropped, contrast-and-luminance adjusted, as well as distorted by noises. Robustness and invisibility against distortions caused by image processing and printing are the essential properties for image watermarking to be applicable in real-world applications. The existing watermark methods used in printed images are less than satisfactory in imperceptibility and robustness. In this paper, the properties of the discretized, printed image and rescanned image in both the spatial and frequency domains are analyzed. Based on these properties, image complexity and template matching theories, this paper proposes a steganography approach for data-hiding in printed image. First, the message to be hidden is coded by using $\mathrm{BCH}$ to facilitate the extraction of hidden information. Secondly, based on appropriate image block partition, the local image complexity of the block is calculated to decide whether to embed watermark in this block. Watermark is embedded on the mid-frequency spectrum in DFT domain. Thirdly, two structural template lines are added in the polar coordinate system for detecting any combination of the geometrical distortions. Then, the hidden data can be extracted without using the original image. The experimental results indicate that the proposed method is robust against standard image manipulation operations, basic attacks and image publishing system. And the robustness and the invisibility of watermark are also satisfactory in printed images.
\end{abstract}

Keywords: DFT, image complexity, information hiding, printed image, steganography, template matching, watermark.

\section{INTRODUCTION}

The printing is used for image reproduction and distribution, mainly including offset printing, relief printing, gravure printing and screen printing. It is popular to transform images between the digital format and the printed pictures. Steganography is a method for secretly delivering information by hiding it in something else (such as image, video, audio and so on), which is one of the main methods for copyright protection, anti-counterfeiting and forensics of presswork now. Nowadays, two methods are used for hiding information in printed image: the special inks (such as fluorescent security inks, infrared (IR) security inks and so on) and the digital watermark. The drawbacks to the former are expensive and unsafe. The latter is effective technology being developed in which copyright information and security information are embedded into the cover image in a way that is robust to a variety of intentional or unintentional attacks. It could protect both digital image and printed one. However, most of previous research on steganography focused on the digital image. After a digital image is printed and rescanned, several attacks are produced, including two fields: geometric

\footnotetext{
*Address correspondence to this author at the Faculty of Electronic Information and Electrical Engineering, Dalian University of Technology, Dalian, Liaoning Province 116024, P.R. China; Tel: +86 13604083663; Fax: +86 041186323736; E-mail: wcyvivien@ 126.com
}

distortion (scaling, rotation, cropping and so on) and changes in pixel values (luminance, contrast and chrominance variations) [1-4]. It was not clear how the printing process affects the image, and how a steganography system can survive it. Therefore, it is very necessary that for copyright protection, anti-counterfeiting and forensics or other purposes (bridging printed media and the internet) an effective steganography system should be able to detect or extract copyright and security information or other required data, regardless of the media format of the image.

Some steganography methods have been proposed to solve the printing and rescanning problems. Especially, steganography algorithms robust to the geometrical distortions (including rotation, scaling, translation, cropping/shearing, projective transformation) have been the focus of research. Several approaches to this problem including exhaustive search, synchronization pattern/template, invariant domain and implicit synchronization using image features are widely used. J. O' Ruanaidh and T. Pun [5] suggested first a watermarking method based on the log-polar map of the Fourier coefficients, known as the Fourier-Mellin Transform. They proposed that the watermark data can be embedded in Discrete Fourier Transform (DFT) magnitudes of the FourierMellin coefficients because of their well-known shifting property to rotation, scaling, and translation (RST) distortions. The Fourier-Mellin transform can efficiently solve the problem that is produced by uniform scaling as well as crop- 


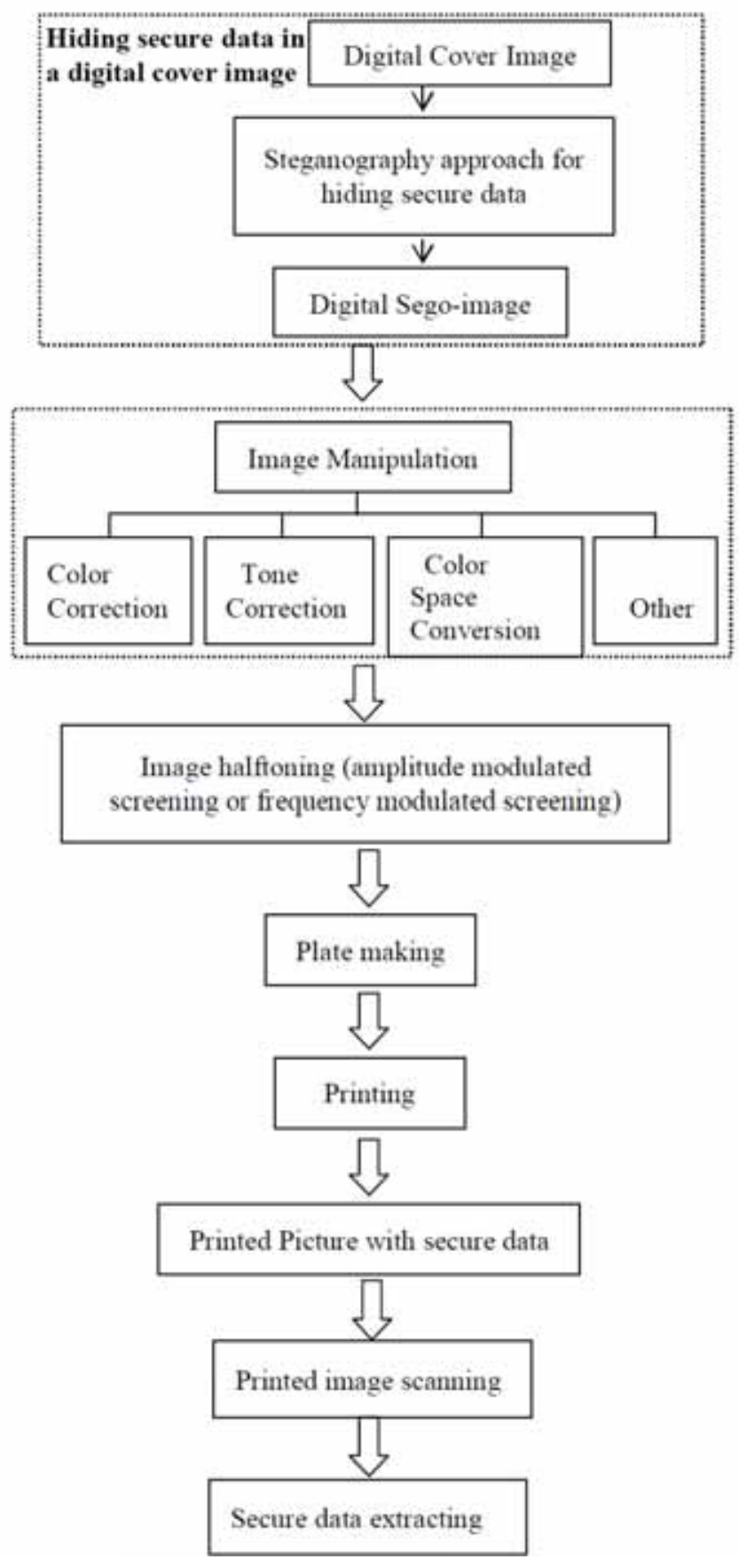

Fig. (1). A steganography scheme in printed image.

ping which maintains the original aspect ratio. However, if the image is cropped with arbitrary aspect ratio as in the printing and rescanning process, this method would become invalid. In $[1,6]$, the Fourier-Mellin-based watermarking method has been discussed in detail and an RST resilient watermarking method has been proposed. Another method proposed by Pereira et al. [7] embeds a registration pattern as well as a watermark into an image. This is an effective solution but can have the problems of reducing the fidelity and tampering the watermark. Solanki [8] studied the printscan process and noted that the low and mid frequency coefficients are preserved much better than the high frequency ones in Fourier transform magnitudes. Yun Chung Chiu [9] presented that a watermark is embedded in a host image as coefficient value peaks that are circularly and symmetrically distributed in middle band of DFT domain. However, since these algorithms do not consider the effect of human vision system (HVS), their performances are not good in keeping visual inconspicuousness. In addition, no algorithms are effective for the traditional printing methods, such as offset printing, intaglio printing, relief printing and so on.

In Section 2 of this article, we analyze the properties of printed pictures in both the spatial and frequency domains; in Section 3, we introduce the fundamental theories and techniques used for watermarking; in Section 4, we introduce our steganography method; in Section 5, we perform experiments and discuss the experimental results; and in Section 6, we conclude the article.

\section{PROPERTIES OF PRINTED IMAGE}

As illustrated in Fig. (1), a steganography scheme in printed image consists of several processes: hiding secure data in a digital cover image, manipulation operations of digital image, image halftoning, plate making, printing, scanning printed image and extracting secure data.

After a digital image is printed and rescanned, the geometric boundary is generally distorted and the pixel values are changed.

\subsection{Distortions of the Geometric Boundary}

The distortion of the geometric boundary in the printing and scanning processes is caused by rotation, scaling, and cropping. Especially, in scanning process the geometric distortion could not be adequately modeled by the well-known rotation, scaling, and translation effects, because of the design of today's Graphic User Interface (GUI). The scanned image may only cover a part of the original picture and may have an arbitrary cropped image size. In [2], several properties of the change of DFT coefficients after geometric distortions are discussed. In cases where DFT size equals image size, rotation in the spatial domain results in the same rotation in the frequency domain. However, if the rotated image is cropped, the center of DFT domain will not be rotated, while other coefficients are rotated, as shown in Fig. (2).

\subsection{Distortions of the Pixel Values}

Since printer is binary device, a digital continuous tone image must be converted to halftone image to print by screening way. A halftone image is represented as a twodimensional array of image elements (pixel), each having a one bit binary value of one or zero. In printing or displaying such an image, each binary value corresponds to the presence or absence of a signal, such as a black or a white dot, shown by Fig. (3). In color halftone images, each pixel location has a vector of 1 bit binary values, where the elements in the vector correspond to color components like red, green and blue (RGB), cyan, magenta, yellow and black (CMYK), etc. In printing, CMYK color mode is always used, where each pixel has one or more multilevel color values (one for grayscale, four typically for color). Halftone processes convert multilevel per pixel images at a source resolution to 

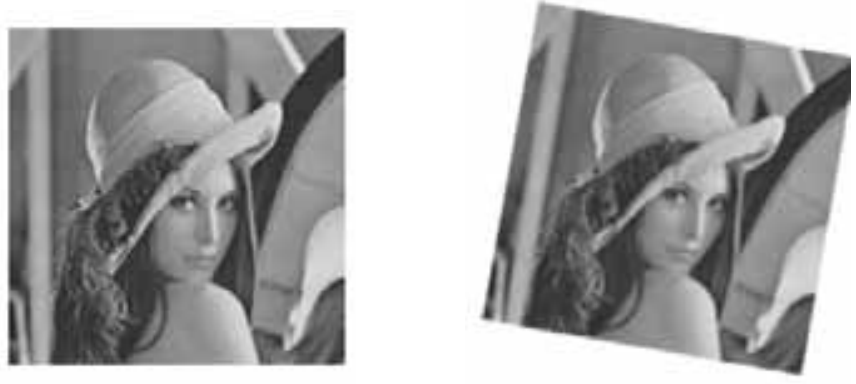

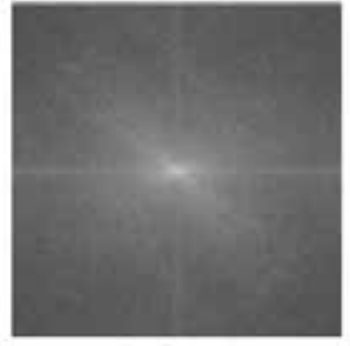

(a) Original
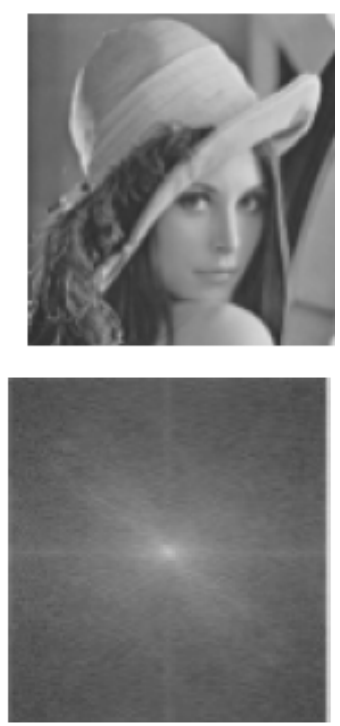

(c) cropped

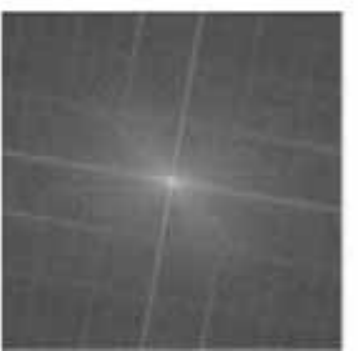

(b) rotated
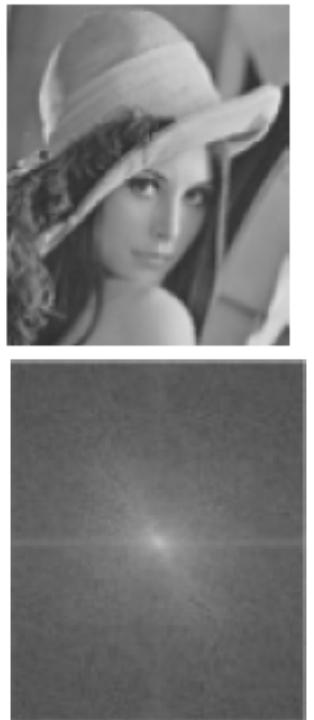

(d) Rotated-and-Cropped

Fig. (2). The spectrum of rotated image, cropped image, rotated-and-cropped image.

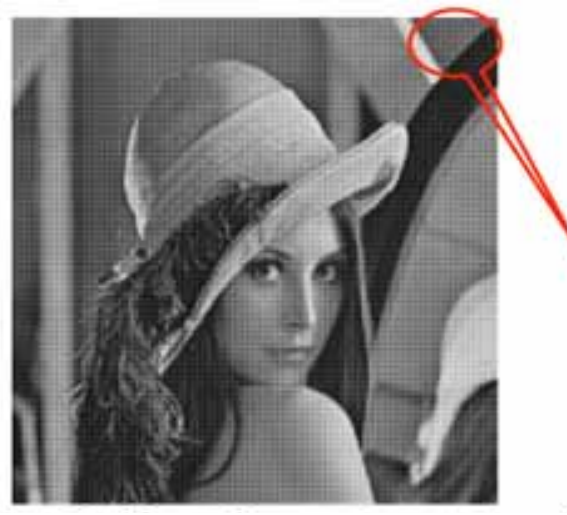

(a) Halftone image

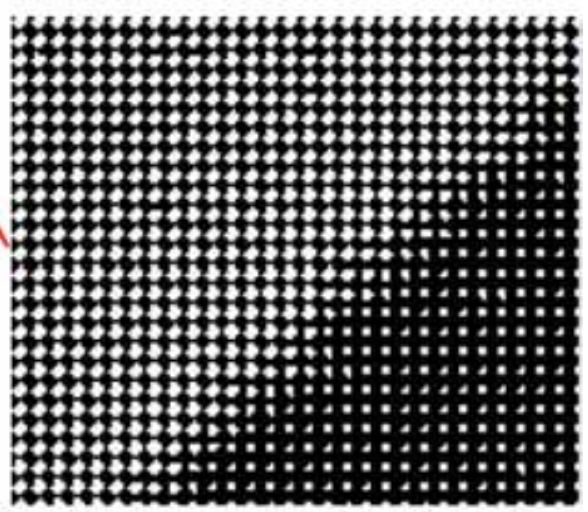

(b) microstructure of halftone image

Fig. (3). The halftone image and its microstructure. 

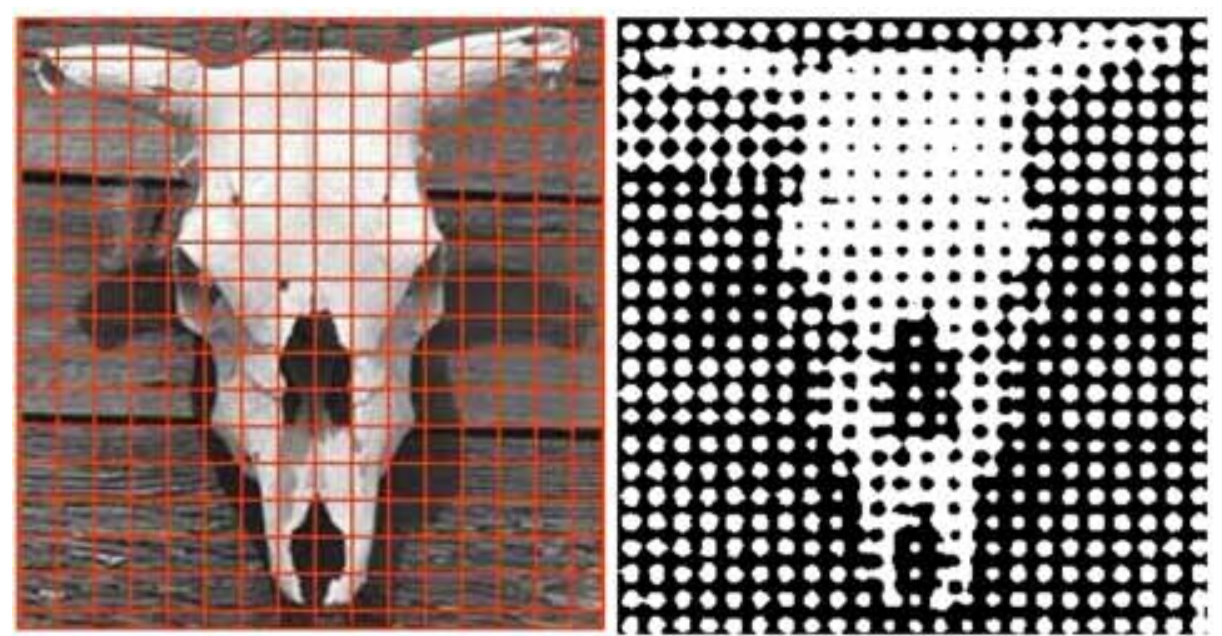

(a) Halftone process

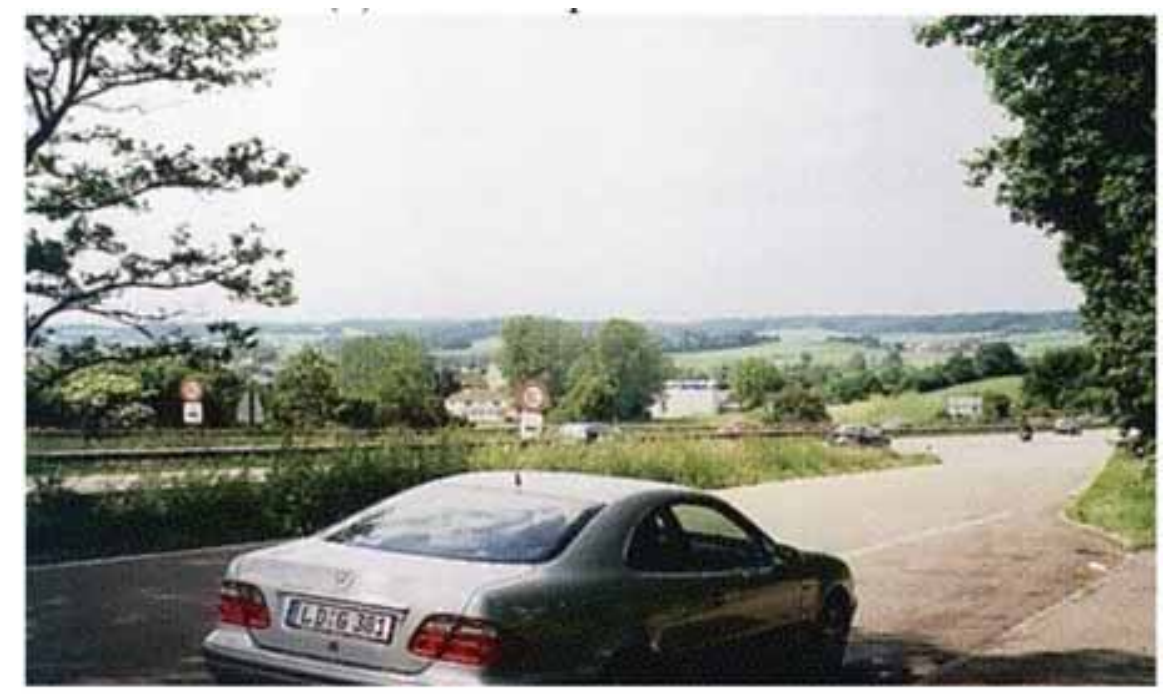

(b) The digital color image

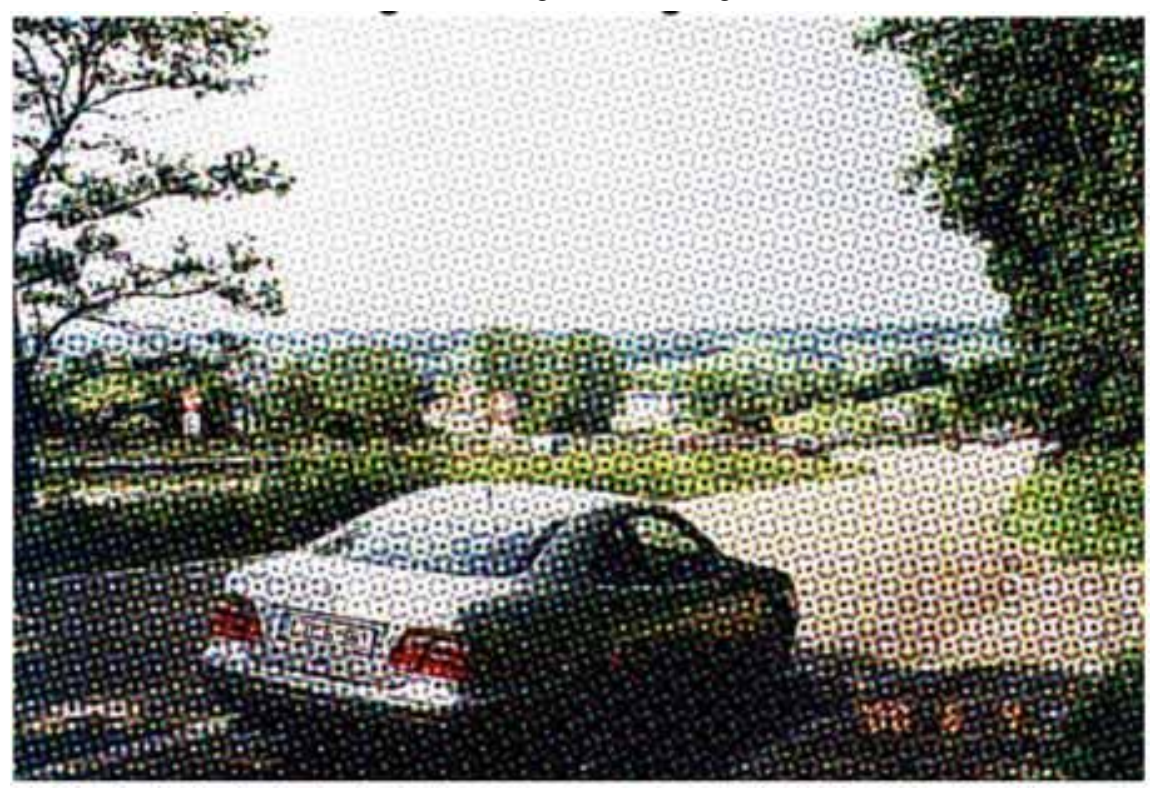

(c) The printed image (screen line 100 lpi)

Fig. (4). Halftone process, printed halftone image with CMYK color components. 


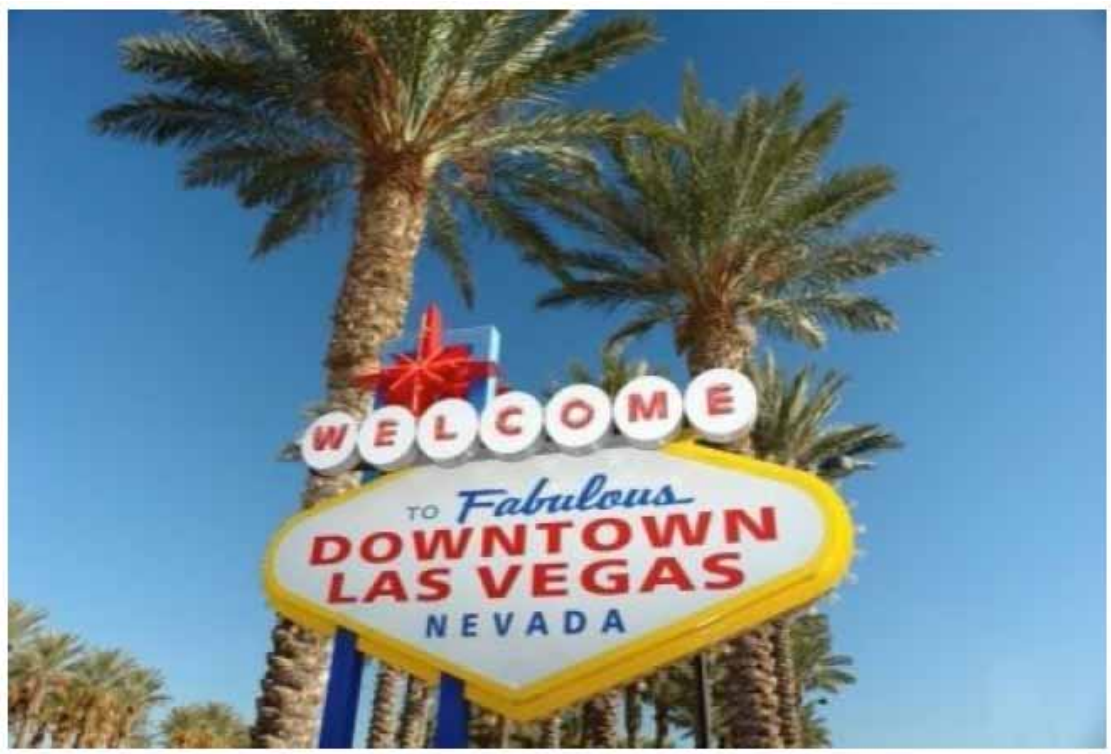

(a) The digital image

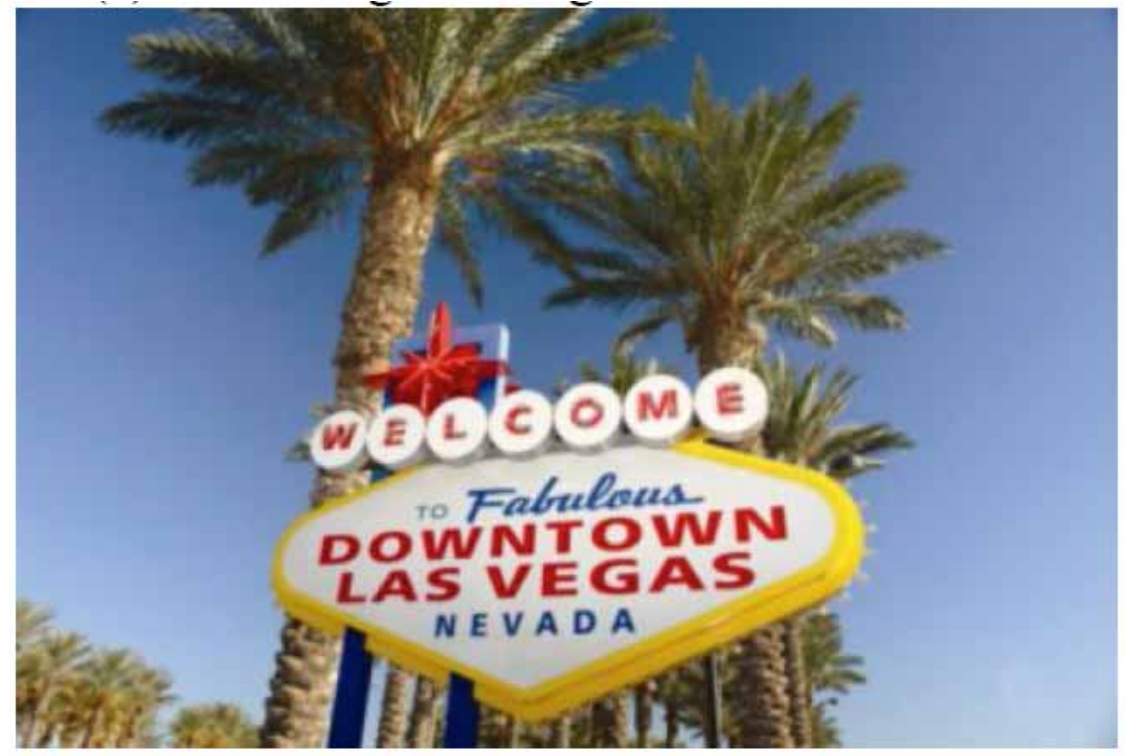

(b) The printed and rescanned image

Fig. (5). The distortion of pixel values.

halftone images at a higher target resolution by subdividing each two-dimension (2D) region representing a location of a multilevel pixel image in a rectangular array into a 2D array of halftone pixel locations and selectively setting bits of the halftone locations to one or zero to represent the value of the multilevel pixel, shown in Fig. (4). In [10], the detailed discussion can be found.

There are a variety of halftone processes. One popular technique used in newsprint, packaging print, business print, etc. for example, is to represent multilevel pixel values as $2 \mathrm{D}$ geometric primitives, comprised of contiguous clusters of halftone pixel dot (called amplitude modulated screening $(\mathrm{AM})$ ). The correct screening parameters which include screen line, screen angle, screen shape and dot area coverage should be chosen to keep the visual quality of image. In tra- ditional color printing, 133 lines per inch (lpi), 150 lpi and 175 lpi screen are chosen; $0^{\circ}, 15^{\circ}, 45^{\circ}$ and $75^{\circ}$ screen angles are employed; and screen shape is circular or rectangular. The digital image shown in Fig. (5a) has been halftoned with the above-mentioned screen parameters, printed, and scanned, as shown by Fig. (5b).

The distortion in pixel values is caused by image halftoning, ink features [11], dot gain [12] in printing and image vision feature changes (including the luminance, contrast, chrominance variations, etc.) in scanning, shown by Fig. (5). The spectrum of image after pixel values distortions is changed nearly.

Thus, the main problem is to solve the geometric distortion. In this paper, we employ the template to correct the geometric distortion. 


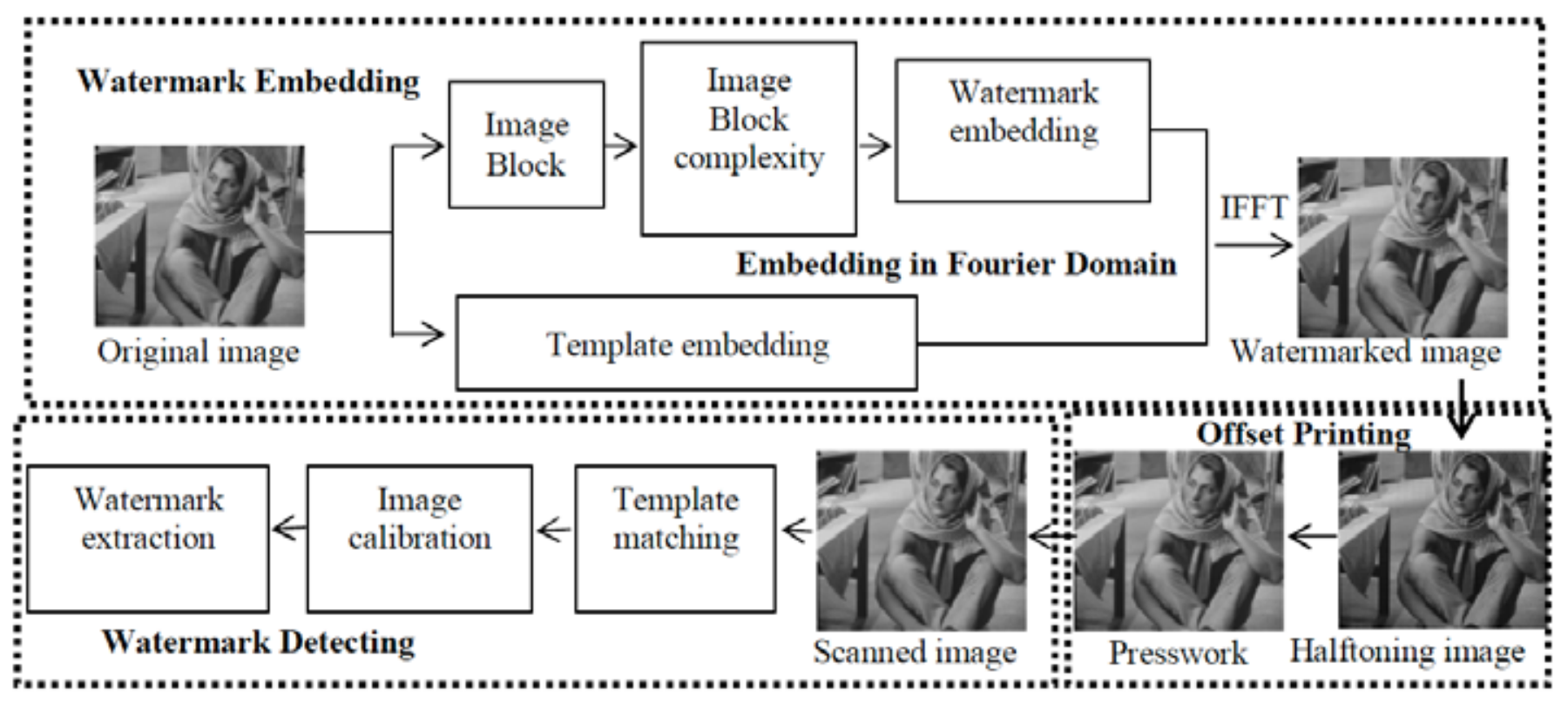

Fig. (6). Information hiding and extracting scheme in presswork.

\section{IMAGE COMPLEXITY AND TEMPLATE MATCH- ING}

\subsection{Image Complexity}

Images, depending on their content, have different complexities. However, a single image may be classified in different complexity classes by different viewers. But there is a global agreement in classifying images by complexity. According to the theory about HVS model and the method for automatically determining the perceptual importance of different regions in an image proposed by Wilfried Osberger [13], there are 5 primary factors influencing image complexity, including contrast, intensity, edginess and texture. Image block complexity of an image is given by Eq. (1).

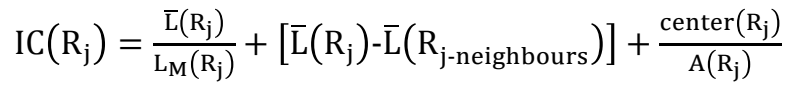

Where $\operatorname{IC}\left(R_{j}\right)$ is the complexity of image block $R_{j}, \bar{L}\left(R_{j}\right)$ is the mean grey level of region $\mathrm{R}_{\mathrm{j}}, L_{M}\left(\mathrm{R}_{j}\right)$ is the maximum grey level of region $\mathrm{R}_{\mathrm{j}}, \overline{\mathrm{L}}\left(\mathrm{R}_{j-\text { neighbours }}\right)$ is the mean greylevel of all of the neighboring regions of $\mathrm{R}_{\mathrm{j}}$, center $\left(\mathrm{R}_{j}\right)$ is the number of pixels in region $\mathrm{R}_{\mathrm{j}}$ which are also in the center $25 \%$ of the image, $A\left(\mathrm{R}_{j}\right)$ is the area of region $\mathrm{R}_{\mathrm{j}}$ in pixels, $b p\left(\mathrm{R}_{j}\right)$ is the number of pixels in the region $\mathrm{R}_{\mathrm{j}}$ which border with other regions, borderpixel $\left(A\left(\mathrm{R}_{j}\right)\right.$ is the number of pixels in region $\mathrm{R}_{\mathrm{j}}$ which also border on the image, and tot_borderpixel is the total number of image border pixels.

\subsection{Template Matching}

The template contains no information but is merely a tool used to extract transformation parameters in the image. Generally, the template consists of a random arrangement of peaks in the Fast Fourier Transformation (FFT) domain [7]. In paper [7], the experimental results showed that using tem- plates of approximately 8 points works best. So in this paper, the template consists of two lines embedded on the midfrequency spectrum in DFT domain. Each line contains eight points. The distance of the adjacent two points is equal. The angles between two lines are controlled by secret key. Template embedding is decided by Eq. (2).

NewMagT $=$ LocalMean $(\mathrm{i})+\omega 1 * \mathrm{~S}(\mathrm{i})+\omega 2 *$ std, $\mathrm{i}=1, \ldots, 8$

Where, NewMagT is the final DFT magnitudes, LocalMean(i) is the mean magnitude of the neighboring 120 points, S(i) is the standard deviation of the neighboring 120 points, std is the total standard deviation of DFT spectrum, and $\omega_{1} *$ and $\omega_{2} *$ are intensity quotients.

\section{PROPOSED STEGANOGRAPHY APPROACH IN PRINTED IMAGE}

Fourier domain is suitable for robust embedding, but it lacks the necessary local precision for transparency. Therefore, based on image complexity, we embed the informative watermark message (such as logo, owner, serial number, etc.) for transparency. On the other hand, a template is used for re-synchronization after geometric distortions. In watermark detecting process, template matching and image calibration are firstly implemented, then watermark data is extracted. As illustrated in Fig. (6), the proposed Information Hiding scheme consists of two embedding processes: watermark information embedding and template embedding.

\subsection{Secure Data and Watermark Data Embedding}

Firstly, the message to be hidden is coded by using Bose, Ray-Chaudhuri and Hocquenghem $(\mathrm{BCH})$ to facilitate the extraction of hidden information. The watermark signal is also processed by using spread spectrum technology to improve robustness of watermark. Secondly, the original image is segmented into blocks with proper size and the image 


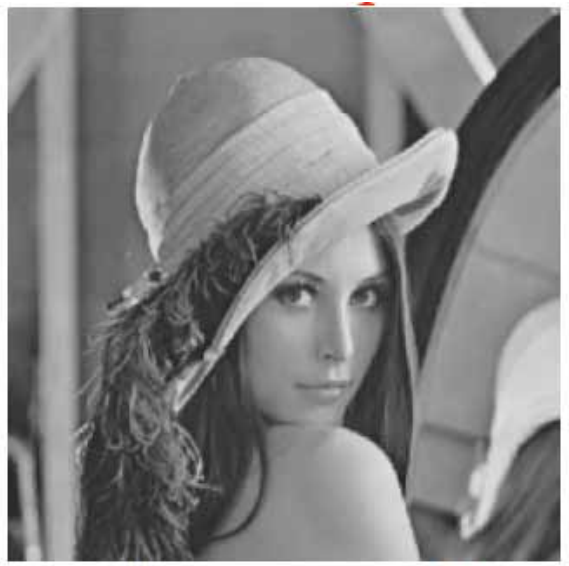

(a) Lena

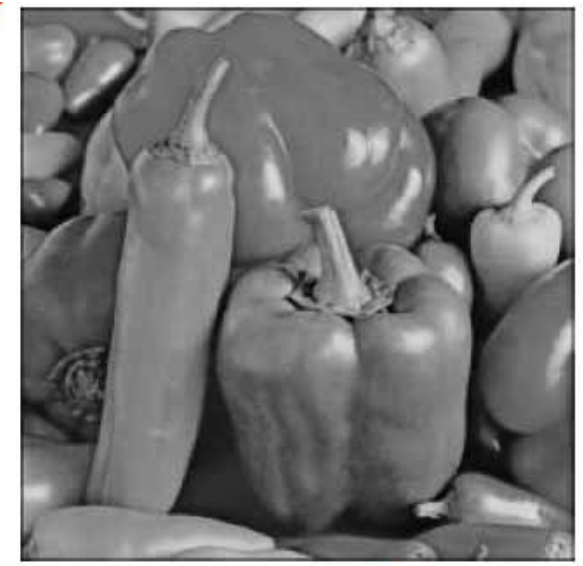

(b) peppers

\section{CY}

(c) $\log 0$
Fig. (7). Cover images and secure information.

complexity of every block is calculated to decide whether to embed watermark data. Thirdly, in this paper, DFT domain is chosen to embed a series of string watermark by one bit in one block according to the image block complexity (calculated by Eq. (1)). Two positions with the same radii $r_{1}$, and $r_{2}$ and different angles $\theta_{1}$ and $\theta_{2}$ are chosen in the middle band. A peak in one position will be created according to the watermark data bit which is 1 or 0 , shown by Eq. (3).

$$
\text { NewMag1 }=\text { Mag1 }+\mathrm{a} * \text { MeanMag, } \mathrm{b}=1
$$

$$
\text { NewMag2 = Mag2 }+\mathrm{a} * \text { MeanMag, } \mathrm{b}=0
$$

Where, NewMag1 and NewMag2 are the adjusted magnitudes after adjustment of two different positions. Mag1 and Mag2 are the magnitudes before adjustment of two different positions. If the watermark bit to be embedded is 1 , the magnitude of the first position will be changed; otherwise, the magnitude of the second position will be changed. a is an intensity factor, and MeanMag is the average magnitude of the block except the zero frequency which is the maximum value. And meanwhile, the symmetric location will also be adjusted.

In order to re-synchronize the watermark after geometric distortions, a structured template is also embedded in Fourier domain as noted above. The embedding strength is determined adaptively by the local mean and standard deviation of Fourier coefficients. In order to avoid visible artifacts in the spatial domain, the embedding strength is minimized to local average value of coefficients plus two standard deviations in [3, 14].

\subsection{Watermark Detection}

After the presswork is scanned, the watermark detection is started which consists of two phases. First, the template detector will try to detect the embedded template. If a template is found, the watermarked image will be recovered from the undergone geometric distortions. After that, another watermark detection process will be performed on the adjusted image.

In watermark extraction, the adjusted image is first divided into blocks with the same size as embedding and the image complexity of sub-block is calculated. If the image com- plexity of sub-block satisfies the condition, the sub-block will be translated to DFT transformation and frequency-shift transformation. The peak-to-average value in two positions is calculated with the same radii $r_{1}$, and $r_{2}$ and different angle $\theta_{1}, \theta_{2}$ as embedding. The watermark bit will be extracted by the following Eq. (4):

$$
\begin{aligned}
& \mathrm{b}=1, \frac{\text { Magp } 1}{\text { NewMeanMag }}>\text { threshol } \\
& \mathrm{b}=0, \frac{\text { Magp } 2}{\text { NewMeanMag }}>\text { threshold }
\end{aligned}
$$

Where, Magp1 and Magp2 are the magnitudes of the two different positions, respectively. NewMeanMag is the average value of all the magnitudes except the zero frequency which is the maximum value in this block.

\section{EXPERIMENTS AND RESULTS}

All experiments have been carried out on a single color offset printer. The amplitude modulated screening (AM) has been used for halftoning image. All algorithms have been implemented in MATLAB system. A color scanner and a computer-to-plate machine have been employed.

The cover images are $1024 * 1024$ gray scale Lena and peppers (showed by Fig. 7a and 7b); besides, the $32 * 32$ logo image is shown by Fig. (7c). The cover images are segmented into $128 * 128$ blocks to choose embedding positions by calculating the image complexity. The blocks with the image complexity value above 0.2 are embedded data to have a better visual quality. In order to evaluate imperceptibility of the algorithm, Peak signal to Noise Ratio (PSNR) between original and stego-image after printing and scanning is computed. In order to compare the extracted and original logo, the bit error rate (BER) and the normalized correlation coefficient (NCC) are calculated. The Bit Error Rate is the ratio of the number of erroneous detected bits to the total number of watermark bits. The normalized cross correlation coefficient is used to measure the similarity between the original and the extracted data.

PSNR $=10 \times \log _{10}\left[\frac{\left(2^{n}-1\right)^{2}}{\text { MSE }}\right]$ 

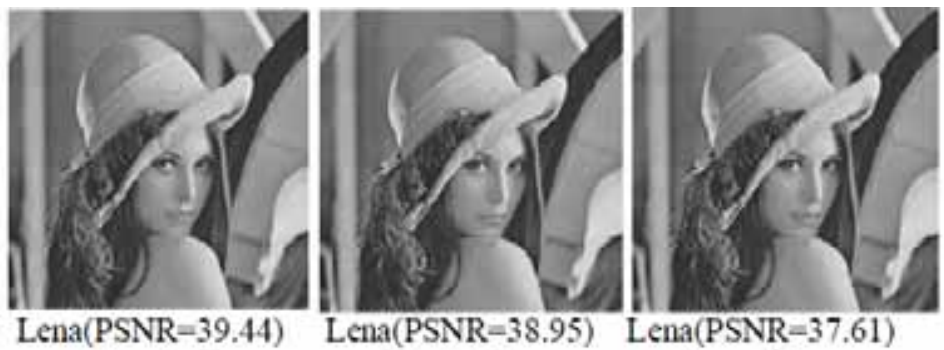

Lena(PSNR=37.61)
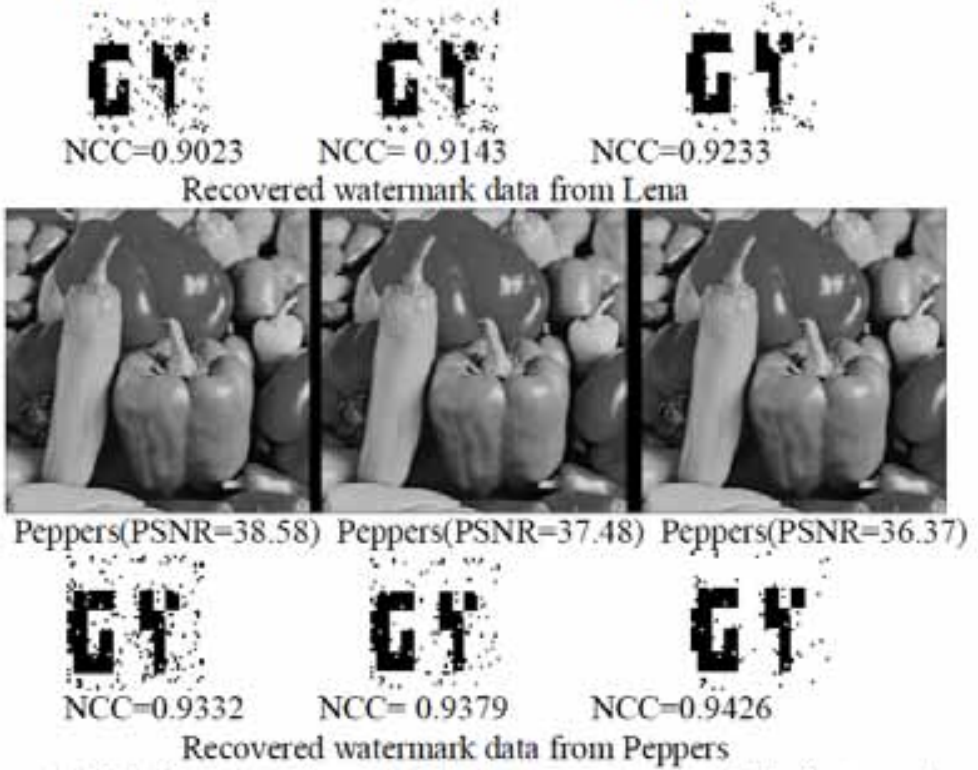

$\begin{array}{lll}\text { (a) Gain factor } \mathrm{a}=4 & \text { (b) Gain factor } \mathrm{a}=6 & \text { (c) Gain factor } \mathrm{a}=8\end{array}$

Fig. (8). Stego-image after printing and scanning, extracted logo with the different gain factors.

Table 1. PSNR, BER and NCC for different cover and secret images for different gain factors.

\begin{tabular}{|c|c|c|c|c|}
\hline Cover images & $\begin{array}{c}\text { Gain } \\
\text { Factors(a) }\end{array}$ & PSNR & BER & NCC \\
\hline \multirow[t]{2}{*}{ Lena } & 6 & 38.95 & 0.052 & 0.9143 \\
\hline & 8 & 37.61 & 0.047 & 0.9233 \\
\hline \multirow[t]{2}{*}{ Peppers } & 6 & 37.48 & 0.038 & 0.9379 \\
\hline & 8 & 36.37 & 0.032 & 0.9426 \\
\hline
\end{tabular}

Where MSE is Mean Square Error between original and print-scanned stego-image.

$$
\mathrm{BER}=\frac{1}{\mathrm{~N}}\left(\sum_{\mathrm{i}=0}^{\mathrm{N}-1} \mathrm{w}(\mathrm{i}) \oplus \mathrm{w}^{\prime}(\mathrm{i})\right) * 100 \%
$$

Where w(i) and w'(i) are the original watermark and the extracted watermark, respectively. $\mathrm{N}$ is the watermark length.

$$
\mathrm{NCC}=\frac{\sum_{\mathrm{i}=1}^{\mathrm{M}} \sum_{\mathrm{i}=1}^{\mathrm{N}}\left(\mathrm{w}_{\mathrm{ij}}-\overline{\mathrm{w}}\right)\left(\mathrm{w}_{\mathrm{ij}}^{\prime}-\overline{\mathrm{w}}^{\prime}\right)}{\sqrt{\sum_{\mathrm{i}=1}^{\mathrm{M}} \Sigma_{\mathrm{i}=1}^{\mathrm{N}}\left(\mathrm{w}_{\mathrm{ij}}-\overline{\mathrm{w}}\right)^{2}} \sqrt{\sum_{\mathrm{i}=1}^{\mathrm{M}} \Sigma_{\mathrm{i}=1}^{\mathrm{N}}\left(\mathrm{w}_{\mathrm{ij}}^{\prime}-\overline{\mathrm{w}}^{\prime}\right)^{2}}}
$$

Where $w$ and w' are the original watermark and the extracted watermark, respectively. $\overline{\mathrm{w}}$ and $\overline{\mathrm{w}^{\prime}}$ are the mean val- ues of the original watermark and the extracted watermark, respectively.

\subsection{The Different Gain Factors}

In this test, the parameters of halftoning image have been chosen: screen line $150 \mathrm{lpi}$, screen angle $0^{\circ}$, screen shape circular. The different gain factors (a) affect the visual quality of stego-image and the correctness of extracted secure data. Based on the stego-image quality and extracted correctness, we test three different gain factors: $\mathrm{a}=4,6,8$. Fig. (8) and Table 1 show PSNR, BER and NCC for different cover and secret images for different gain factors. 

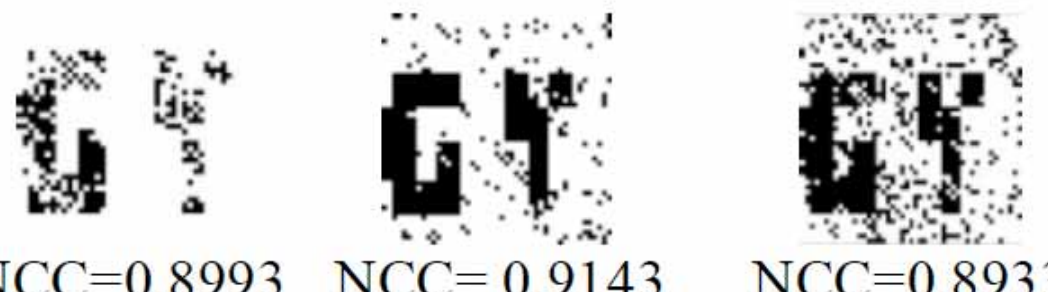

$\mathrm{NCC}=0.8993 \quad \mathrm{NCC}=0.9143 \quad \mathrm{NCC}=0.8933$

Recovered watermark data from Lena
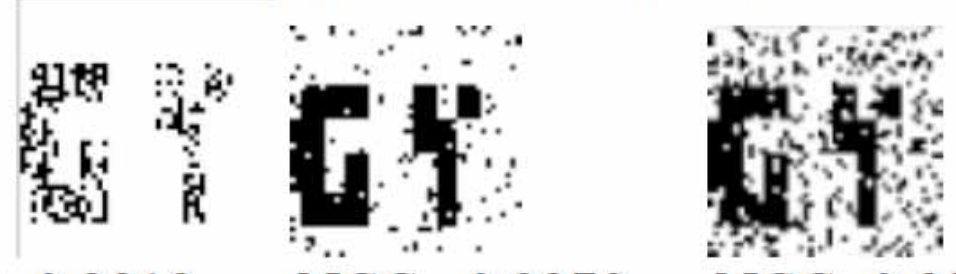

$\mathrm{NCC}=0.8912$

$\mathrm{NCC}=0.9379$

$\mathrm{NCC}=0.8946$

Recovered watermark data from Peppers

\section{(a) Threshold $=2$ (b) Threshold $=3$}

(c) Threshold $=4$

Fig. (9). Extracted logo with the different thresholds.

Table 2. BER and NCC for different cover and secret images for different gain factors.

\begin{tabular}{|c|c|c|c|}
\hline Cover Images & Thresholds & BER & NCC \\
\hline \hline \multirow{2}{*}{ Lena } & 2 & 0.084 & 0.8993 \\
\cline { 2 - 5 } & 3 & 0.052 & 0.9143 \\
\cline { 2 - 5 } & 4 & 0.097 & 0.8933 \\
\hline \multirow{2}{*}{ Peppers } & 2 & 0.092 & 0.8912 \\
\cline { 2 - 5 } & 3 & 0.038 & 0.9379 \\
\cline { 2 - 5 } & 4 & 0.082 & 0.8946 \\
\hline
\end{tabular}

This experimental result shows that the PSNR values are greater than $36 \mathrm{~dB}$ for all cover images for different gain factors. Thus, this proposed algorithm is effective for offset printing and is good in terms of invisibility of the embedded data.

\subsection{The Different Thresholds}

The different thresholds mainly affect the correctness of extracted secure data. Fig. (9) and Table 2 show the results of extracted data with the different extracted thresholds (Threshold $=2,3,4$ ). In this test, the gain factor a is 6 . The parameters of halftoning image have been chosen: screen line $150 \mathrm{lpi}$, screen angle $0^{\circ}$, and screen shape circular.

Table 2 shows when the gain factor is 6, the extraction threshold $\mathbf{3}$ should be chosen.

In the following, the results of evaluating the proposed data hiding algorithm for robustness against a range of common screening and scanning attacks and geometric distortion are presented.

\subsection{The Screening Parameters}

Because the printer is a binary device, image must be halftoned to print. In this paper, AM screening method has been employed, which is most popular in the traditional printing. The main parameters include screen line, screen angle, screen shape and dot area coverage, among which the first two are more important for the image quality.

\subsubsection{The Different Screen Lines}

In traditional offset print, 133 lpi, 150 lpi, 175 lpi and 200 lpi screen are used. The higher the screen line, the better the printed image quality. In this test, the gain factor a is 6 , the extraction threshold 3 , screen angle $0^{\circ}$, and screen shape circular. Lena and peppers images embedded secure data are halftoned with the above-mentioned screen lines respectively, then printed and scanned. Finally, the secure data is extracted from rescanned image. The results are shown in Fig. (10) and Table 3. 
Table 3. PSNR, BER and NCC for different cover and secret images for different screen lines.

\begin{tabular}{|c|c|c|c|c|}
\hline Cover Images & Screen Line (lpi) & PSNR & BER & 0.079 \\
\hline \hline \multirow{3}{*}{ Lena } & 133 & 36.44 & 0.052 & 0.8892 \\
\cline { 2 - 5 } & 150 & 38.95 & 0.059 & 0.9143 \\
\cline { 2 - 5 } & 175 & 37.21 & 0.065 & 0.9033 \\
\cline { 2 - 5 } & 200 & 36.98 & 0.068 & 0.8913 \\
\cline { 2 - 5 } & 133 & 36.58 & 0.038 & 0.9379 \\
\cline { 2 - 5 } & 150 & 37.48 & 0.046 & 0.9126 \\
\cline { 2 - 5 } & 175 & 37.17 & 0.051 & 0.8993 \\
\hline
\end{tabular}
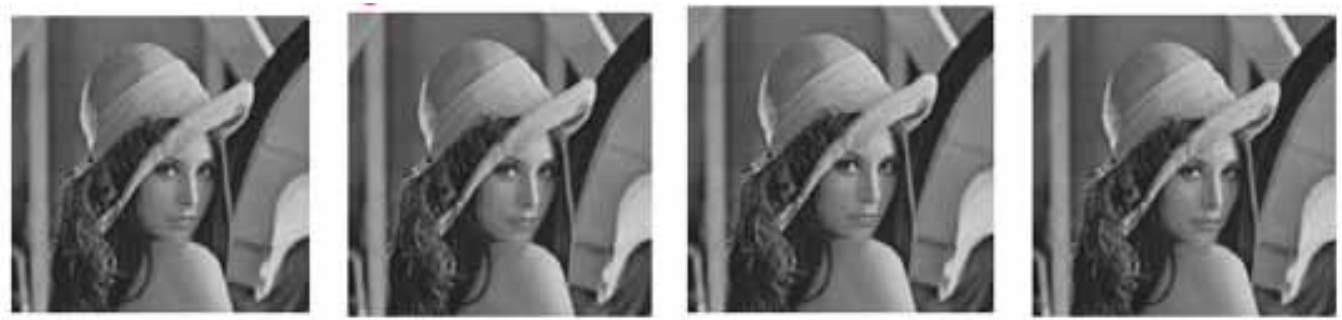

screen line $=1331$ pi screenline $=1501$ pi screenline $=1751$ pi screen line $=2001$ pi
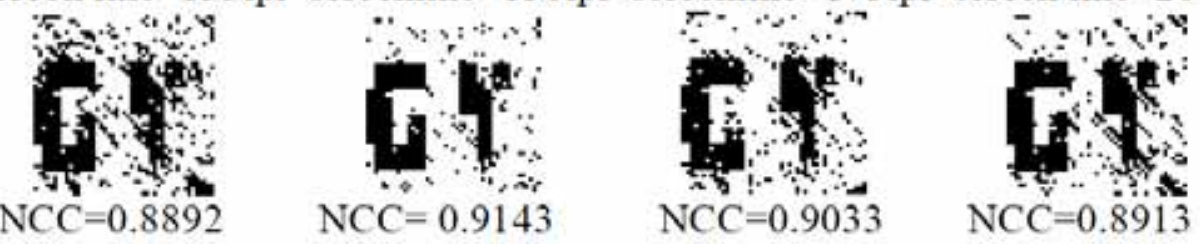

Recovered watermark data from Lena
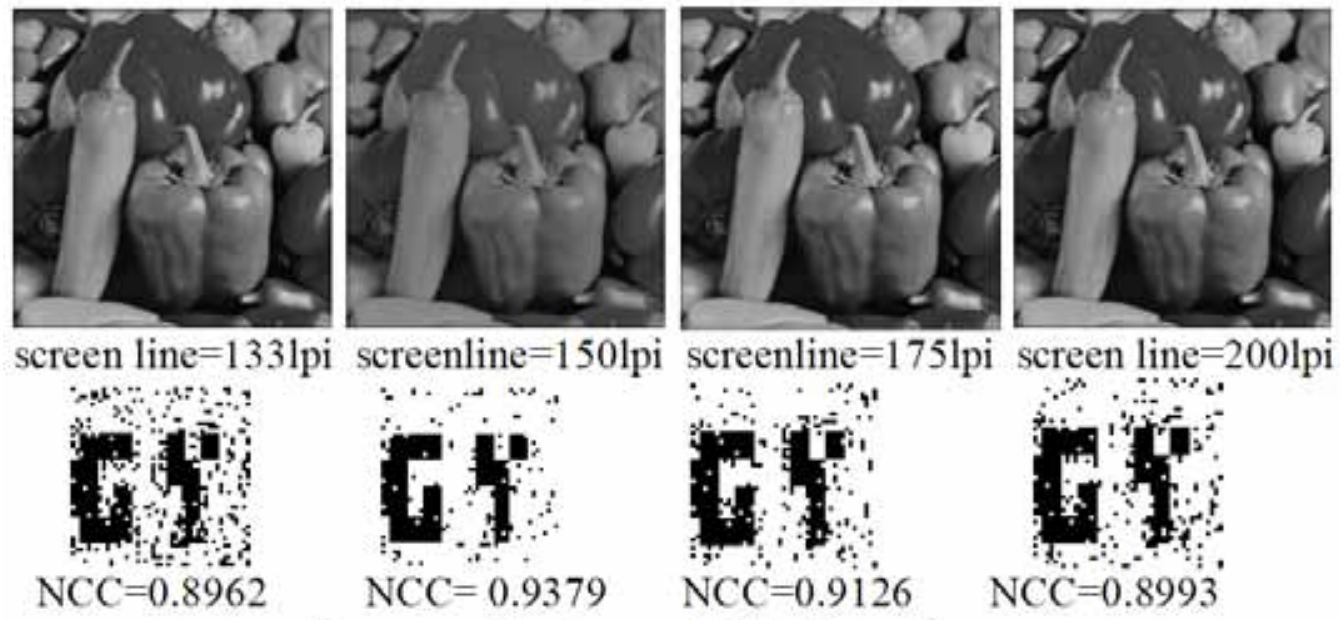

\section{Recovered watermark data from Peppers}

Fig. (10). Stego-image after printing and scanning, extracted logo with the different screen lines.

This experimental result shows when the screen line is 150 lpi, PSNR values and NCC are greater, and BER is smaller. We have found when the screen line is closer to the resolution ratio of the cover image, the extracted data is more correct.

\subsubsection{The Different Screen Angles}

$0^{\circ}, 15^{\circ}, 45^{\circ}$ and $75^{\circ}$ screen angles are often employed in print. In this test, Lena and Peppers images are halftoned with the four screen angles respectively, then printed and 
Table 4. PSNR, BER and NCC for different cover and secret images for different screen lines.

\begin{tabular}{|c|c|c|c|c|}
\hline \multirow{3}{*}{ Cover Images } & Screen Angle $\left(^{\circ}\right)$ & PSNR & BER & 0.052 \\
\hline \hline \multirow{3}{*}{ Lena } & 0 & 38.95 & 0.065 & 0.9143 \\
\cline { 2 - 5 } & 15 & 37.97 & 0.081 & 0.8943 \\
\cline { 2 - 5 } & 45 & 35.67 & 0.065 & 0.8683 \\
\cline { 2 - 5 } & 75 & 37.98 & 0.038 & 0.9943 \\
\cline { 2 - 5 } & 0 & 37.48 & 0.064 & 0.89379 \\
\cline { 2 - 5 } & 15 & 36.87 & 0.089 & 0.8606 \\
\hline
\end{tabular}
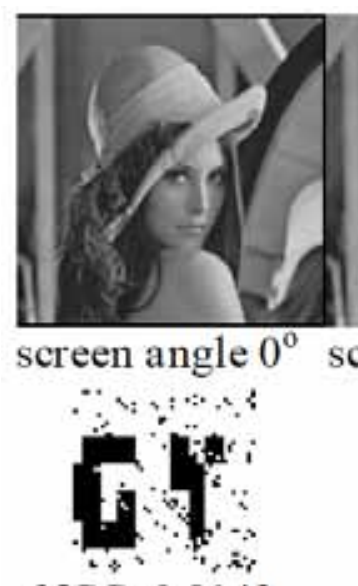

$\mathrm{NCC}=0.9143$

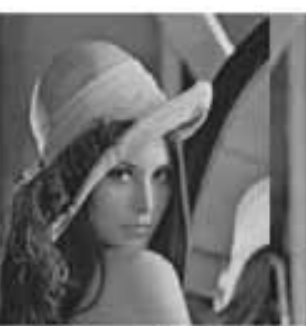

ser

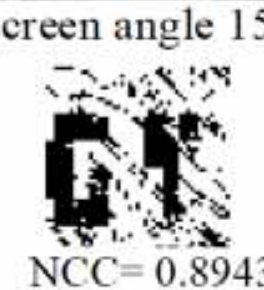

Recovered watermark data from Lena

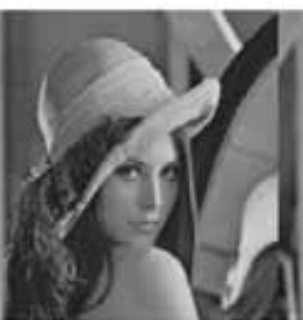

screen angle $45^{\circ}$

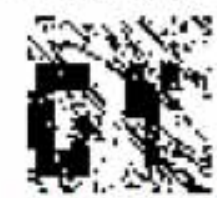

$\mathrm{NCC}=0.8683$

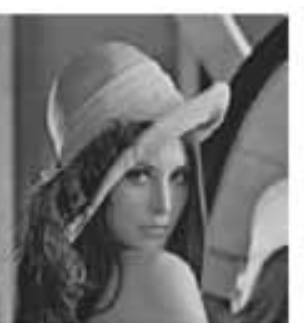

screen angle $75^{\circ}$

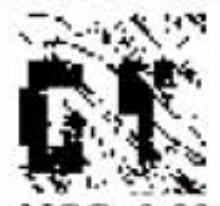

$\mathrm{NCC}=0.8943$
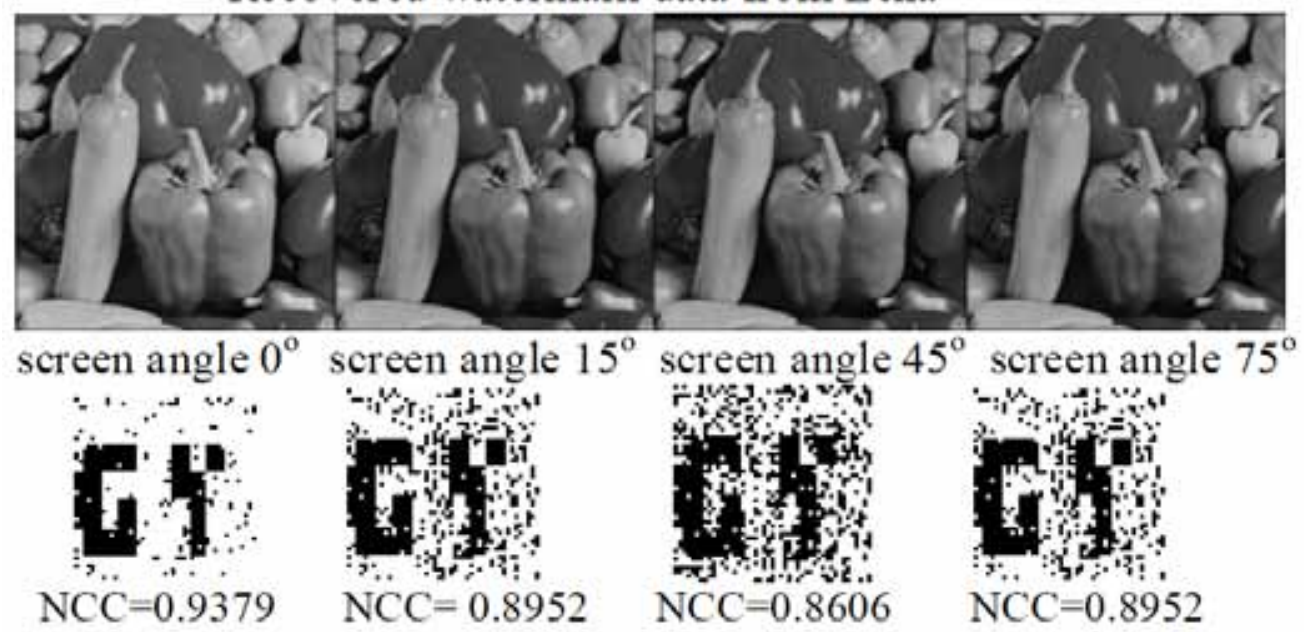

Fig. (11). Stego-image after printing and scanning, extracted logo with the different screen angles.

scanned. Finally, the secure data is extracted from rescanned image. Screen line is $150 \mathrm{lpi}$. Other parameters are the same as above. The results are shown in Fig. (11) and Table 4.

Fig. (11) and Table 4 show when the screen angle is $0^{\circ}$, PSNR values and NCC are greater, and BER is smaller.
When screen angles are $15^{\circ}$ and $75^{\circ}$, the extracted logos are same, and when screen angle is $45^{\circ}$, the correctness of extracted data is worst. In the grayscale print, screen angle is often $0^{\circ}$. While in the color print, screen angle of black is often $45^{\circ}$, black component is not fit for embedding secure data. 
Table 5. BER and NCC for different cover and secret images for cropping attack.

\begin{tabular}{|c|c|c|c|}
\hline Cover Images & Rotated $\left(^{\circ}\right)$ & BER & NCC \\
\hline \hline \multirow{3}{*}{ Lena } & 5 & 0.095 & 0.8516 \\
\cline { 2 - 4 } & 10 & 0.081 & 0.8703 \\
\cline { 2 - 4 } & 15 & 0.072 & 0.8899 \\
\hline \multirow{2}{*}{ Peppers } & 0 & 0.097 & 0.8434 \\
\cline { 2 - 4 } & 15 & 0.086 & 0.8702 \\
\cline { 2 - 4 } & 45 & 0.077 & 0.8862 \\
\hline
\end{tabular}
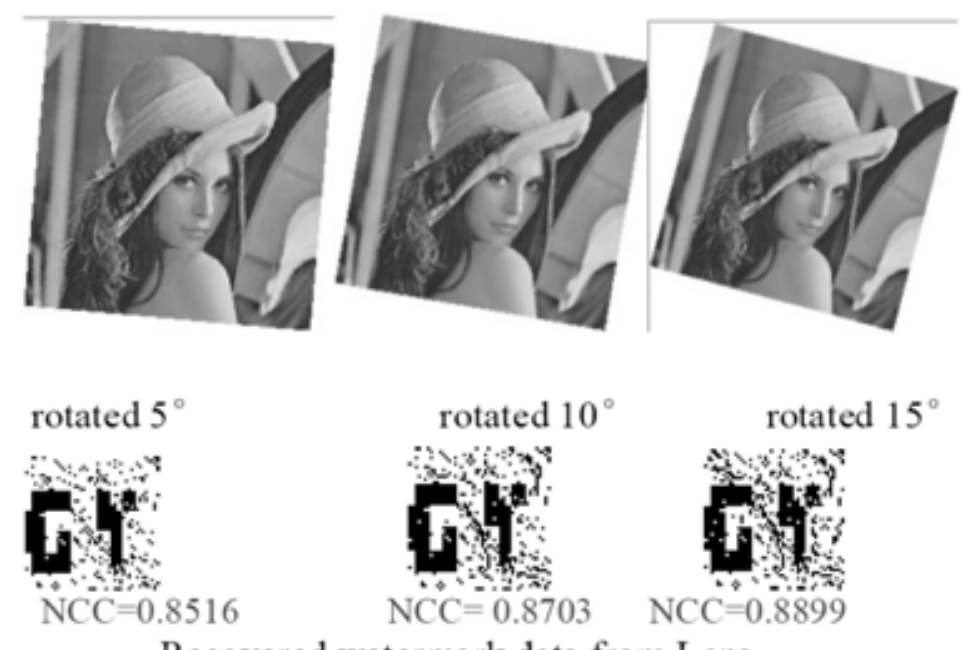

Recovered watermark data from Lena

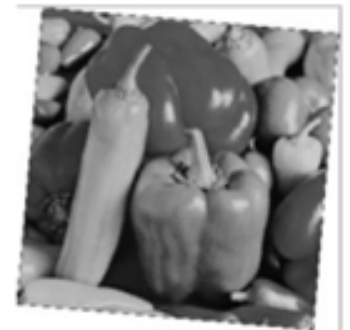

rotated $5^{\circ}$

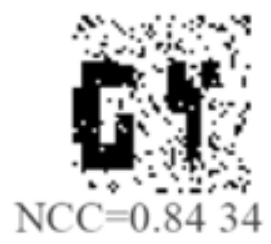

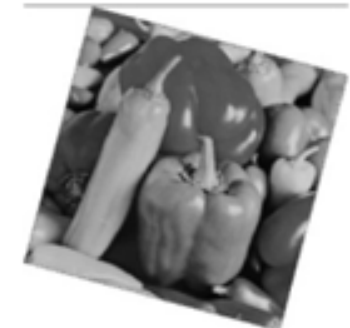

rotated $10^{\circ}$

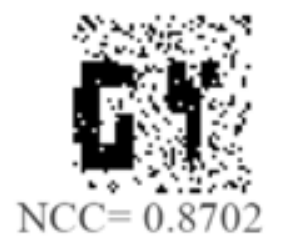

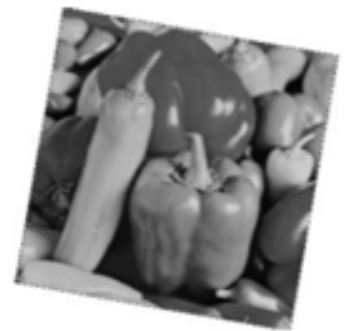

rotated $15^{\circ}$

Fig. (12). Cropped stego-image after printing and scanning, extracted logo with the different screen angles.

\subsection{The Rotated Operation}

Lena and Peppers images are tested for geometric attack of rotating by varying rotating angle from $5^{\circ}$ to $15^{\circ}$. And the result is listed in Table 5. The extracted images, Lena image and Peppers image after rotating $5^{\circ}, 10^{\circ}$, and $15^{\circ}$, are shown in Fig. (12). From the obtained results, with the growth of rotating angle, it is found that the smaller the rotation angle is, the poorer the correctness of the extracted data is. But the secure data can be extracted because of using template matching technology in our algorithm.

\subsection{The Cropped Operation}

Lena and Peppers images are tested for geometric attack of cropping by varying cropping percentage from 5\% to $20 \%$. And the result is listed in Table 6. The extracted images, 
Table 6. BER and NCC for different cover and secret images for cropping attack.

\begin{tabular}{|c|c|c|c|}
\hline Cover Images & Cropped (\%) & BER & NCC \\
\hline \hline \multirow{3}{*}{ Lena } & 5 & 0.060 & 0.8915 \\
\cline { 2 - 4 } & 10 & 0.066 & 0.8823 \\
\cline { 2 - 4 } & 15 & 0.074 & 0.8783 \\
\cline { 2 - 4 } & 20 & 0.068 & 0.8702 \\
\hline \multirow{3}{*}{ Peppers } & 0 & 0.069 & 0.8875 \\
\cline { 2 - 4 } & 10 & 0.067 & 0.8852 \\
\cline { 2 - 4 } & 15 & 0.078 & 0.8746 \\
\cline { 2 - 4 } & 20 & 0.070 & 0.8681 \\
\hline
\end{tabular}

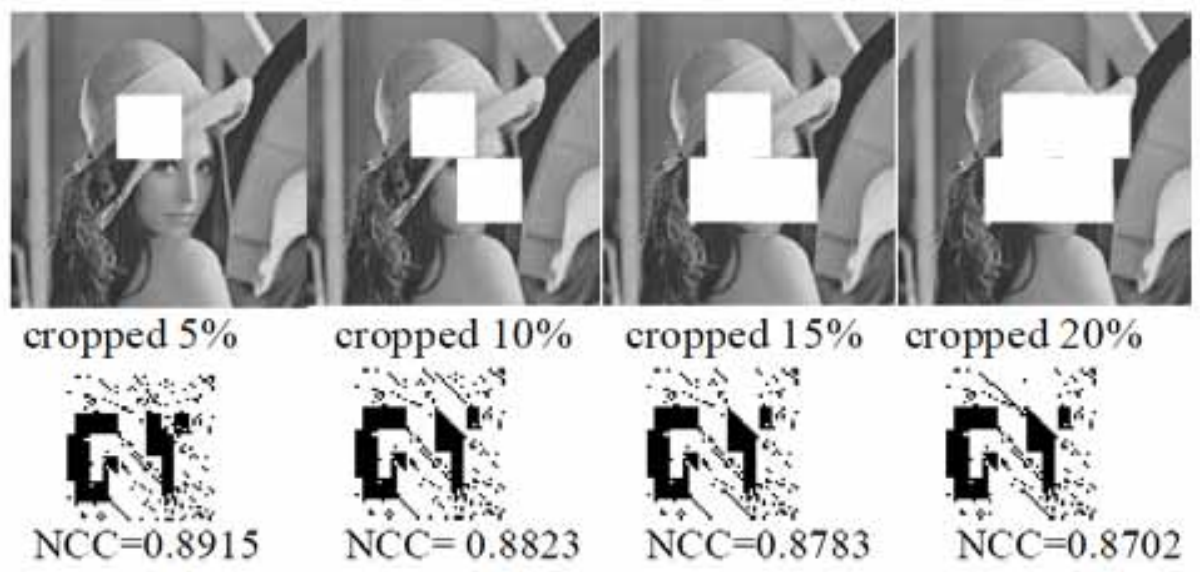

Recovered watermark data from Lena
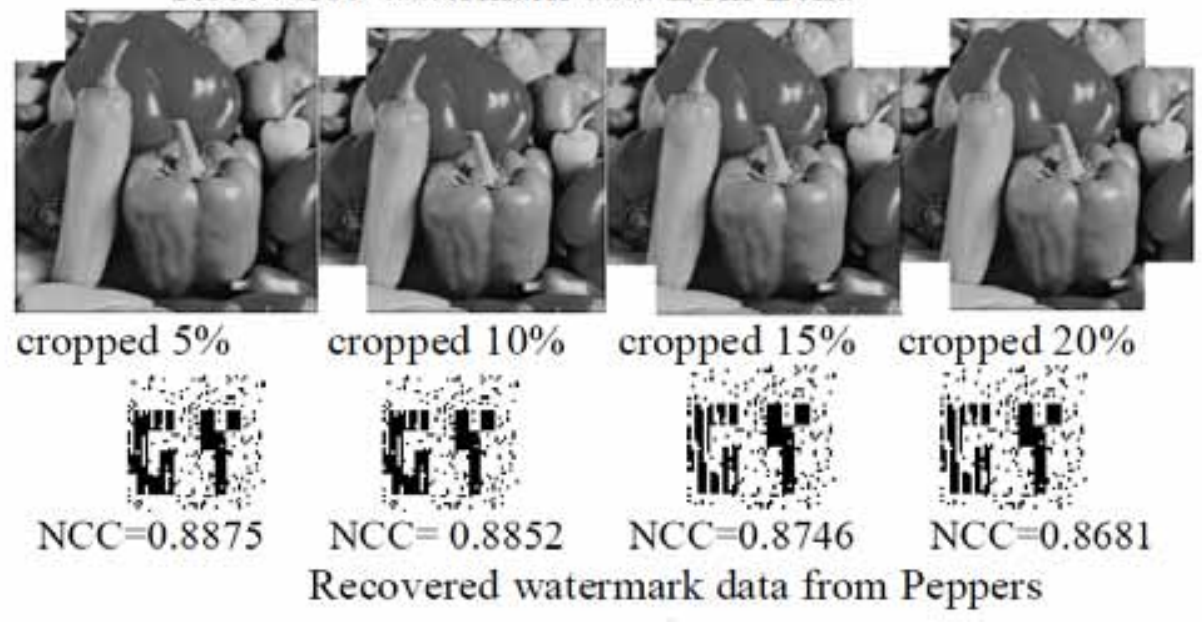

Fig. (13). Cropped stego-image after printing and scanning, extracted logo with the different screen angles.

Lena image after cropping $5 \%, 10 \%, 20 \%$ and $25 \%$ middle portion, and Peppers image after cropping 5\%, 10\%, 20\% and $25 \%$ edge portion, are shown in Fig. (13). From the obtained results, the correctness of the extracted data is decreased; it can be detected and extracted.

\section{CONCLUSION}

In this paper, we presented a method to embed a watermark message into an image utilizing image complexity and template matching technology in DFT transform domain. Based on the properties of the printed image coefficients in 
the Fourier domain, watermark information is embedded in the mid-frequencies. The experimental results show that the proposed algorithm achieves robustness against AM screening, printing, scanning and basic geometric attacks. It can be used to protect the presswork copyright of the owner, anticounterfeiting and forensics of presswork. Performance for robustness and imperceptibility of our proposed algorithm has been evaluated using bit error rate (BER), normalized correlation coefficient (NCC), and peak signal to noise ratio (PSNR) for cover images and secure image. The observed results demonstrate that this proposed algorithm also induces distortion to a certain extent in the cover image. The main features of the algorithm are imperceptibility, robustness and blind watermark recovery. However, this algorithm is not very satisfactory at the small-angle rotation (smaller than $5^{\circ}$ ). The availability of this algorithm has not been tested in other printing methods (such as relief printing, gravure printing and screen printing). So, capacity feature, optimization and application in other printing of the algorithm will be our next research. On the other hand, the watermark algorithm for color printed image will be also our next consideration.

\section{CONFLICT OF INTEREST}

The authors confirm that this article content has no conflict of interest.

\section{ACKNOWLEDGEMENTS}

Declared none.

\section{REFERENCES}

[1] C.Y. Lin, M. Wu, J.A. Bloom, I.J. Cox, M.L. Miller and Y.M. Lui, "Rotation, scale, and translation resilient watermarking for images”, IEEE Transition Image Processing, vol. 10, no.5, pp. 767-782, 2001.
[2] C. Guo, G. Xu, X. Niu, Y. Yang, and Y. Li, "A color image watermarking algorithm resistant to print-scan, In: International Conference on Wireless Communication, Networking and Information Security(WCNIS2010) June 25-27, Beijing, China, 2010.

[3] L. Huajian, and S. Martin, "Improved fourier domain patchwork and template embedding using spatial masking," Electronic Imaging, vol. 8303, pp. 1-13, 2012.

[4] Q. Liu, "An adaptive blind watermarking algorithm for color image", Telkomnika, vol. 11, no. 1, pp. 302-309, 2013.

[5] J. O'Ruanaidh and T. Pun, "Rotation, scale and translation invariant spread spectrum digital image watermarking," Signal Processing, vol. 66, no. 3, pp. 303-317, 1998.

[6] C.Y. Lin, M. Wu, M.L. Miller, I.J. Cox, J. Bloom and Y.M. Lui, "Geometric distortion resilient public watermarking for images," submitted to SPIE Security and Watermarking of Multimedia Content II, San Jose: CA, Jan. 2000.

[7] S. Pereira, S and O Ruanaidh, "Template based recovery of Fourier-based watermarks using Log-polar and Log-log maps", Proceedings of ICMCS, vol. 1, pp. 870-874, 1999.

[8] K. Solanki, U. Madhow, B.S. Manjunath and I. El-Khalil, "Printscan resilient Data hiding in image", IEEE Transactions on Information Forensics and Security, vol. 1, no. 4, pp. 464-478, 2006.

[9] Y. C. Chiu, and W. H. Tsai, "Copyright protection against printand-scan operations by watermarking for color images using coding and synchronization of peak locations in frequency domain", Journal of information science and engineering, vol. 22, no. 4, pp. 483-496, 2006.

[10] Z. He and C. A. Bouman, "AM/FM halftoning: digital halftoning through simultaneous modulation of dot size and dot density", Journal of Electronic Imaging, vol.13, no. 2, pp. 286-302, 2004.

[11] B. Jablonovský, "The study of offset prin-ting inks properties drying by oxypolymerization", Master Thesis (in Czech), University of Pardubice: Czech Republic, 2010.

[12] Yang, L. "A unifiedmodel of optical and physical dot gain in print color reproduction", Journal of Imaging Science and Technology, vol. 48, no. 4, pp. 347-353, 2004.

[13] W. Osberger, and A.J. Maeder, "Automatic identification of perceptually important regions in an image,"Intemational Conference on Patten Recognition, vol. 1, pp. 701-704, 1998.

[14] S. Siddharth, and T.J. Siddiqui. "A security enhanced robust steganography algorithm for data hiding", International Journal of Computer Science, vol. 3, no. 9, pp. 131-139, 2012.

(C) Wang and Li; Licensee Bentham Open.

This is an open access article licensed under the terms of the Creative Commons Attribution Non-Commercial License (http://creativecommons.org/licenses/by-nc/3.0/) which permits unrestricted, non-commercial use, distribution and reproduction in any medium, provided the work is properly cited. 